A method has been developed to detect hazardous conditions of contaminated air in urban areas in real time for an arbitrary number of pollutants. The method is based on restoring the hidden dynamics of the combined risk of instantaneous action on the basis of the current measurements of the concentration of pollutants at the point of control. Other data on current conditions at the point of control are not used in the developed method. Therefore, the method, in contrast to known analogs, is universal and can be applied for arbitrary conditions and control points. At the same time, the restored dynamics of the level of the combined risk of instantaneous action makes it possible not only to identify dangerous conditions relating to contaminated atmospheric air but, on the basis of the current recurrence of combined risk levels, to assess the probability of detecting and predicting dangerous levels in the combined risk of instantaneous action in real time at the predefined point of control. Using the developed method at several control points in an arbitrary area would make it possible to determine the space-time distribution of the levels of the combined risk of instantaneous action of atmospheric pollution on the population within a territory. Experimental measurements of the concentration of formaldehyde, ammonia, and nitrogen dioxide in the atmosphere have been performed at the point of control within an industrial city with an air pollution level of 37 units on the AOC scale (USA). Based on the measurements, the method has been confirmed to be feasible. It was established that at the time of a credible dangerous event, the level of the combined risk of instantaneous action was approximately $10^{-3}$ with a single probability of this level. This level of the combined risk is about $10^{5}$ times higher than the corresponding upper limit of permissible individual risk. It is shown that the maximum forecast time of the dangerous level of combined risk under the considered conditions does not exceed 18 hours

Keywords: dangerous air pollution, checkpoint, current concentrations of pollutants, combined risk, recurrence plot
DOI: $10.15587 / 1729-4061.2020 .213892$

\section{DEVELOPMENT OF A METHOD FOR} DETECTING DANGEROUS STATES OF POLLUTED ATMOSPHERIC AIR BASED ON THE CURRENT RECURRENCE OF THE COMBINED RISK

\author{
B. Pospelov \\ Doctor of Technical Sciences, Professor* \\ V. Kovrehin \\ Doctor of Technical Sciences, Associate Professor \\ Research Center** \\ E. R y b k a \\ Doctor of Technical Sciences, Senior Researcher \\ Research Center** \\ E-mail: rybka@nuczu.edu.ua \\ O. Krainiuk ov \\ Doctor of Geographical Sciences, Associate Professor \\ Department of Environmental Safety and Environmental Education \\ V. N. Karazin Kharkiv National University \\ Svobody sq., 4, Kharkiv, Ukraine, 61022 \\ O. Petukhova \\ $\mathrm{PhD}$, Associate Professor \\ Department of Fire Prevention in Settlements** \\ T. B u t e n ko \\ $\mathrm{PhD}$, Senior Researcher* \\ P. B o r o d y c h \\ $\mathrm{PhD}$, Associate Professor \\ Department of Fire and Rescue Training** \\ I. Morozov \\ $\mathrm{PhD}$, Senior Researcher*** \\ O. Horbov \\ $\mathrm{PhD}$ \\ Military Institute for Tank Troops \\ National Technical University "Kharkiv Polytechnic Institute" \\ Poltavskiy Shlyah st., 192, Kharkiv, Ukraine, 61098 \\ I. Hrachova \\ $\mathrm{PhD} * * *$ \\ *Department of Organization and Coordination of Research Activities \\ Scientific-Methodical Center of Educational Institutions in the Sphere of Civil Defence \\ O. Honchara str., 55a, Kyiv, Ukraine, 01601 \\ **National University of Civil Defence of Ukraine \\ Chernyshevska str., 94, Kharkiv, Ukraine, 61023 \\ $* * *$ Department of Research and Organization \\ National Academy of the National Guard of Ukraine \\ Zakhysnykiv Ukrainy sq., 3, Kharkiv, Ukraine, 61001
}

Copyright (c) 2020, B. Pospelov, V. Kovrehin, E. Rybka, O. Krainiukov, O. Petukhova, T. Butenko, P. Borodych, I. Morozov, O. Horbov, I. Hrachova Accepted date 07.10 .2020

\section{Introduction}

The main danger for the sustainable development of civilization in the 21st century is the increased strategic risks. An analysis of the dynamics of these risks reveals that the leading place belongs to the risk to human life [1]. At the same time, one of the main risk factors for health is considered to be air pollution with harmful substances (AP). In this regard, the tools and methods that allow the real-time detection of dangerous conditions of the contaminated atmosphere, as well as preventing their occurrence, in order to prevent disruption of regular human life activities, are of particular relevance. This is due to the need to employ these tools and methods in the information and control systems to monitor and manage security in urban areas experiencing AP, as well as in the elimination of accidents and emergencies related to AP. 


\section{Literature review and problem statement}

Study [2] proposed a method to assess the risk of adverse effects on humans at accidental pollution, based on a combination of CFD (computational fluid dynamics) technology and a risk assessment method based on doses of pollutants. The use of a given method requires a large amount of $a$ priori information of a local nature, which, under actual conditions, is not usually known and can change over time. In addition, this information depends on the location site. For this reason, the overall computational complexity, and the required time resource to implement the method, do not make it possible to use it to quickly identify risk to populations in different regions. This demonstrates the expediency of developing methods to detect hazardous contamination based on an assessment of the current risk that can be used for different regions of the world. The results from a study into assessing the impact of short-term AP are reported in [3]. The study is based on different models and methods. In this case, GIS-based scattering models were used as baseline models, interpolations between monitoring areas, regression and optimization models, as well as the models of linear or spatial scattering of pollution plumes. Two main approaches are currently used to mitigate or eliminate the negative impact of AP on humans. The first one is based on the quantification of AP concentration. The second one employs methods of assessing the risk of negative effects on humans, inflicted by AP [4]. A method of accounting for the dynamic activity of sources of air pollution is considered in [5] in order to assess the dynamic impact on the population. In this case, the study is limited only to the consideration of particulate matter in AP. Other characteristic types of AP and their combined effect are not considered. Study [6] focuses on the issue of low concentrations of AP. It is noted in [6] that the low concentrations of AP may be associated with a significant risk of disruption to a person's normal life, and their peak concentrations may even lead to the risk of death [7]. It is noted in [6,7] that current meteorological factors and the amount of pollutants emitted have a significant impact on the level of AP. The importance of meteorological factors is that they can contribute to the accumulation of harmful substances in the atmosphere, as well as prolong undesirable chemical reactions with the formation of secondary, more dangerous, pollutants. At the same time, it is noted in [8] that wind, air temperature, as well as precipitation and fog, exert the largest influence. Current concentrations of harmful substances in the atmosphere in cities are usually monitored at certain checkpoints or stationary control points. This makes it possible to track the real dynamics of concentrations of harmful substances in time and space. In general, the transfer, dispersal, and accumulation of pollutants in the atmosphere are quite complex processes of a non-linear nature. Therefore, for AP processes, the linearity principle is not usually obeyed [9], which leads to the incorrect use of most known methods and models that describe the actual physical processes relating to AP. However, it is noted in [10] that in reality the rapid detection of dangerous AP states and public health risks is of paramount importance. It is proposed in [11] to treat AP with harmful substances in the form of some complex nonlinearly dynamic system. In this case, the input impacts for such a system should be the emissions of harmful substances from various industrial facilities. To study the dynamics of complex non-linear dynamic systems, as noted in [12], it is better to use a non-linear toolkit, which has a series of applied advantages. At the same time, the basis of this toolkit is the fundamental property of dissipated dynamic systems implying the recurrence of their states (RS) [12]. The non-linear toolkit from [12] makes it possible, based on the RP method, to display any complex dynamics of the state of dynamic systems in the form of an appropriate distribution of points on the plane [13]. The methods from the theory of deterministic and stochastic dynamic systems applied to analyze various natural systems, including the AP systems, are considered in [14]. However, the methods and models obtained are typically described by the systems of deterministic or stochastic differential equations. At the same time, the more complex the system under study, the more equations need to be taken into consideration in the methods and models to adequately describe them. In this case, it is noted in [15] that most natural systems can be explored by methods from the theory of dynamic systems and fractal sets. Paper [16] reviews a method of the rapid detection of dangerous AP in cities based on the application of RPs and proposed RS measures. However, it is noted in [16] that the accuracy of representing the state dynamics involving RP and PC is generally influenced by the type of state space considered, as well as the parametric uncertainty. Thus, the RP method for the case of space with a scalar product is considered in [17]. In this case, the accuracy of representing the state dynamics using RP is only dependent on parametric uncertainty. An adaptive approach is proposed in [18] to overcome the parametric a priori uncertainty of the method reported in [17]. An approach to overcoming the parametric a priori uncertainty, based on the use of an uncertainty function for the current measurable concentrations of AP, is considered in [19]. It is noted that the method of the uncertainty function makes it possible not only to overcome the parametric uncertainty characteristic of the RP method but also to identify dangerous states of AP. However, in the cited works, the detection of dangerous conditions of AP is carried out by methods that do not take into consideration the risk to humans caused by the dangerous conditions of the contaminated atmosphere. A mathematical model is considered in [20] to jointly identify the health risks to the population and detect the dangerous AP conditions in cities. However, the model proposed in [20] is only valid for one arbitrary pollutant and does not detect hazardous AP conditions for a combination of different harmful substances that are normally present in the atmosphere. Therefore, this model is not able to assess the combined health risk from several pollutants. It is noted in [21] that there is sufficient evidence from practical experience about the connection between AP and the violation of human normal life. At the same time, most known publications address methods for examining the link between AP and diseases in the population, usually over large areas around the world. These studies and the methods used are based primarily on the analysis of open data on the concentrations of different pollutants and other necessary parameters, averaged over a rather long time interval (minimum for a year). Therefore, it is not possible to use known methods to detect current hazardous conditions of contaminated air in real time. In this regard, new methods are needed to detect dangerous AP states in real time. An important feature of such methods should be the capability to detect dangerous conditions of contaminated atmosphere and combined risk from pollution. This would make it possible to use such methods in the information-management systems to control and pre-empt the emergence of hazardous 
conditions of AP to manage the risk to the population under a regular operation of industrial facilities polluting the air. At the same time, that could minimize the risk to public health in the aftermath of accidents and emergencies related to AP.

\section{The aim and objectives of the study}

The aim of this work is to develop a method capable of detecting the dangerous conditions of contaminated air in urban areas in real time, as well as to assess the current level of combined risk for the population. The method should also make it possible to predict the emergence of dangerous air pollution conditions in order to devise appropriate organizational and technical measures and management decisions aimed at preventing and minimizing the effects of AP.

To accomplish the aim, the following tasks have been set:

- to develop a method to detect hazardous conditions of contaminated air in urban areas in real time for an arbitrary number of pollutants, as well as to determine the current level of combined risk to the population of these areas;

- to perform experimental measurements of the current concentration for several air pollutants in an actual industrial city, and to test the feasibility of the method based on the results obtained.

\section{Developing a method for detecting hazardous atmospheric conditions for an arbitrary number of pollutants}

The closest prototype to the method being developed is known technology described in [20]. However, this technology does not detect dangerous air pollution in real time for multiple pollutants. This significantly reduces the scope of practical application. In addition, it is not possible to determine the current level of combined risk to the population if there are several pollutants in the air at the same time. The undesirable effects of air pollution on the population are usually characterized by excess concentrations of individual pollutants in terms of their threshold limit value. However, this excess does not say what health risk to the population is inflicted in this case. Therefore, in the world practice of protecting the population from atmospheric pollution, an approach was adopted that is based on health risks. According to this approach, AP is assessed for the risks of chronic and instantaneous action. At the same time, the risk of chronic action usually characterizes the long-term effects of concentrations of one of the pollutants. This means that the risk of chronic action cannot be used to detect dangerous air pollution in real time. The risk of instantaneous action is usually associated with short intervals of exposure to concentrations of pollutants on humans. Therefore, the risk of instantaneous action is chosen as a basic attribute in the developed method for detecting hazardous air conditions in real time.

For the case of one air pollutant, the risk of instantaneous action at the point of measuring the current concentrations $\mathrm{C}_{\mathrm{r}}$ will be determined from the expression given in [22]

$$
R\left(C_{r}\right)=\frac{1}{\sqrt{2 \pi}} \int_{-\infty}^{\alpha+b \lg \left(C_{r} / M P C\right)} \exp \left(-\frac{y^{2}}{2}\right) \mathrm{d} y,
$$

where $\alpha$ and $b$ are the parameters that depend on the toxicological properties of a particular atmospheric pollutant (haz- ard class); $C_{r}$ is the current value of the concentration of the pollutant at the specified point $r, \mathrm{mg} / \mathrm{m}^{3}$; TLV is the threshold limit value of the single concentration of the pollutant, $\mathrm{mg} / \mathrm{m}^{3} ; y$ is an arbitrary variable of integration.

The risk of instantaneous action (1) determines the likelihood of death (disease) for a person experiencing AP from a pollutant of the concentration $C_{r}$. The numerical values of the coefficients $\alpha$ and $b$ in expression (1) are typically determined on the basis of special toxicological studies into the properties of the corresponding pollutants. According to (1), the risk of instantaneous action depends on the current concentration of a particular pollutant, which makes it possible to determine the level of risk in real-time measurements. In practice, however, there are typically several pollutants in the atmosphere. In this case, pollutants can relate to different classes of danger and accept different values of the coefficients $\alpha$ and $b$.

Therefore, if there are the air pollutants, at the predefined measuring point $M$, at different concentrations and belonging to different classes of danger, the combined risk of instantaneous action $R_{t}^{M}$ at time $t$, according to [23], can be represented as follows:

$$
R_{t}^{M}=1-\prod_{m=1}^{M}\left(1-R\left(C_{r}^{m}(t)\right)\right)
$$

where $C_{r}^{m}(t)$ is the current value of the concentration of the $m$-th air pollutant at the $r$-th set point at time $t, \mathrm{mg} / \mathrm{m}^{3}$.

When detecting and pre-empting the emergence of hazardous pollutants based on comparing the measured concentrations of pollutants against their TLV, it is typically necessary to know the current meteorological parameters and characteristics of urban infrastructure in the vicinity of the measured point. However, such a comparison of the current concentrations to TLV makes it possible to identify only the fact of excess or the absence of excess. At the same time, it does not say anything about the level of risk to the population exposed to AP. Therefore, according to the method being developed, it is proposed to restore the dynamics not of the concentration of individual pollutants but the dynamics of the combined risk of instantaneous action. In the future, by analyzing the current dynamics of the combined risk caused by an arbitrary number of pollutants, it will be possible to identify the dangerous AP conditions.

In a general case, the measurement of pollutant concentrations at the designated points within a territory can be carried out both discretely and continuously over time. Under the existing AP control system, measurements are typically performed discretely over time. Therefore, for this case, the combined risk will be determined discretely over time. In this case, each $i$-th discrete value of the level of risk (2) is matched with the discrete level of the combined risk $R_{i}^{M}=R_{t=i}^{M}$. The totality of discrete values of the level of risk $R_{i}^{M}, i=1,2, \ldots, N_{s}$, considered over a limited time interval, will determine the discrete trajectory of the combined risk of instantaneous action, due to an arbitrary number of atmospheric pollutants. To identify the unknown systemic features of the combined risk dynamics, it is proposed to use an RP method, which makes it possible to visually display these hidden features in the form of the distribution of black and white dots on the corresponding plane [24]. In this case, black dots will display the recurrent (close) levels of the combined risk, white dots - the absence of such levels in the observed risk dynamics. The RP-method-based rule of such a 
representation for the discrete values of the levels $R_{i}^{M}$ of the combined risk will be determined from the following ratio

$$
R_{i, j}^{M, \varepsilon}=\Theta \varepsilon\left(-\left|R_{i}^{M}-R_{j}^{M}\right|\right), \quad i, j=1,2, \ldots, N_{S},
$$

where $\Theta()$ is the Heaviside function; $\varepsilon$ is the size of the region of the combined risk level recurrence (CRLR); $N_{s}$ is the size of a sample of the observed levels $R_{i}^{M}$ of the combined risk.

If the hazardous states of AP are present at a controlled point, then, taking into consideration all factors in representation (3), the black dots will correspond to CRLR. The main drawback of rule (3) is low speed. This is explained by the fact that (3) is based on the totality of all data on the level of the combined risk in the observed time interval. Therefore, rule (3) does not make it possible to represent CRLR in real time. This means that it is not possible to use representation (3) to detect real-time hazardous conditions of AP through a combination of harmful substances. Therefore, to use representation (3) in real time, one should impose an additional constraint $i>j$ on the region where the discrete time moments change. In this case, only the combined risk levels that are available at the current time and the preceding moments will be used. Rule (3) subject to the constraint $i>j$ makes it possible to restore the hidden features in the system dynamics, determined by the discrete levels $R_{i}^{M}$ of the combined risk, in real time. In this case, the RP's form has a lower triangular shape of the distribution of black and white dots on the plane. The configuration of the distribution of black and white dots on the plane in this case will visually display the hidden properties of the dynamic system that generates the discrete levels $R_{i}^{M}$ of the combined risk. However, the visual representations obtained in this case as an appropriate RP form do not make it possible to algorithmically identify the features of the properties of the dynamic system, which generates these risks associated with dangerous AP states.

For the algorithmic detection in real time of dangerous AP states, caused by a set of harmful substances, it is proposed to use a window measure of CRLR, similar to [25]. However, according to the proposed method, the measure from [25] is applied to representation (3) considering the restriction $i>j$, and enables a real-time identification of the current measure. Given this, an algorithm for determining the current CRLR window measure for the predefined size $\varepsilon$ of the CRLR vicinity can be presented in the following form:

$$
M_{2}(i, w, \varepsilon)=\text { if }\left(i<\omega,(i+1)^{-1} \sum_{n=0}^{i} R_{i, n}^{M, \varepsilon}, \omega^{-1} \sum_{n=0}{ }^{w-1} R_{i, i-n}^{M, \varepsilon}\right),
$$

where $w$ is the size of a moving averaging window (the number of average discrete values in representation (3) considering $i>j)$.

The numerical values of the current CRLR measure (4) can detect dangerous levels of the combined risk of instantaneous action and the AP state in real time. For example, if the injection of pollutants into the atmosphere occurs at CRLR time points, it may lead to an additional increase in the level of the combined risk to the person inflicted by AP, even causing an instant or premature death.

Thus, the proposed method for detecting hazardous conditions of contaminated air differs from known ones by that it is based on appropriate mathematical ratios whose application makes it possible to determine the current levels of the combined risk of instantaneous action and to restore, based on them, the dynamics of the states of a certain system of AP that generates these levels of the combined risk. Next, the proposed algorithm, based on the restored dynamics of an atmospheric pollution system, makes it possible to detect CRLR in real time, which identifies current hazardous conditions of the contaminated atmosphere at the predefined point of control. Applying the method at several checkpoints within a city makes it possible to identify the space-time distributions of CRLR to public health and dangerous AP conditions, without the use of conventional meteorological information and characteristics of urban infrastructure in the area of control points. The proposed method can also be used in the development of operational organizational and technical measures and when making management decisions in the course of emergency response in order to prevent or reduce the risk of disruption of normal human life caused by air pollution.

\section{Verifying the proposed method using the actual measurements of atmospheric pollutant concentrations as an example}

To test the proposed method, we measured the concentration of three pollutants at the predefined point of control within actual urban infrastructure whose air pollution level was about 37 units based on the AQI scale (USA). When choosing the type of experimental pollutants, we took into consideration the sources of pollution characteristic of urban infrastructure, caused by motor vehicles [26], fires [27, 28], and industrial enterprises [29]. In addition, the relation between pollutants and acid rain and the poisoning of aquifers was taken into consideration [30]. The results of the measurements, the measuring instruments used, the coordinates of the control point, as well as the conditions of the experiment, are described in [16,20]. All measurements were carried out in accordance with the procedure given in [16]. Formaldehyde (hazard class 2, carcinogen), ammonia (hazard class 4), and nitrogen dioxide (hazard class 2) were considered as standard pollutants. The concentrations of these pollutants were measured in $\mathrm{mg} / \mathrm{m}^{3}$. Measurements were repeated 4 times a day $(1.00 ; 7.00 ; 13.00 ; 19.00)$ except for holidays and weekends. A test interval of one month was selected to test the method. During the test interval, air pollution facilities were operating normally. The discrete concentration measurement numbers for the test interval are numbered from 480 to 600 . According to open data, there were neither accidents nor emergencies during the specified test interval. However, at count time 509, according to open data, there was a dangerous event associated with the mass poisoning of people in the open territory.

During the experiment, the measured concentrations of formaldehyde in the atmosphere at the point of control were much smaller than the maximum one-time TLV. Therefore, two pollutants were used to test the method, in the form of nitrogen dioxide and ammonia. As an illustration, Fig. 1 shows the measured concentrations of the pollutants in the form of nitrogen dioxide and ammonia (red dots), as well as their corresponding values of the levels of instantaneous risk (blue points).

In addition, Fig. 1 shows the maximum one-time TLV for the considered pollutants. The figure also demonstrates a recalculated limit level for the upper boundary of acceptable individual risk, equal to $5.70810^{-8}$. Fig. 2 shows, for the case $i>j$, the RP representation (3) for the test interval and for a separate fragment limited to 500 and 515 counts, provided the recurrence neighborhood size is $\varepsilon=0.005$. 


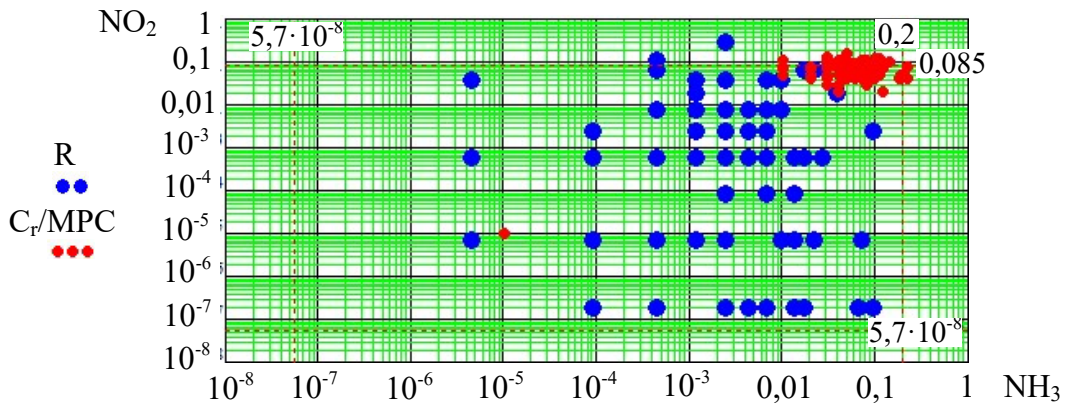

Fig. 1. Experimental data illustrating the measured concentrations of atmospheric pollutants and their corresponding levels of instantaneous risk

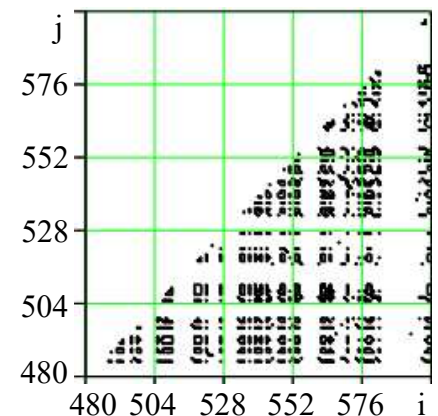

$a$

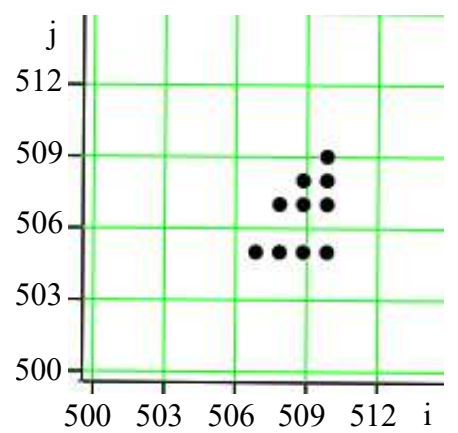

$b$

Fig. 2. RP of the combined risk of instantaneous action (3) for different intervals: $a$-entire test interval; $b$ - fragment of the test interval

As an illustration, Fig. 3 shows the dynamics in the discrete values of the combined risk of instantaneous action (2) and the CRLR window measure (4) on the fragment of the interval from counts 500 to 520 , with the recurrence neighborhood size $\varepsilon=0.01$ for two values $w=1$ and $\omega=0$ (no averaging).

In Fig. 3, marker marks count 509, during which, it is known for sure, there was a dangerous event associated with a clear violation of the conditions for the normal life (loss of consciousness) of people in the open territory. Therefore, this countdown is chosen not only to verify the method but also as a possible assessment of its reliability. It should be noted, however, that it is not possible to detect hazardous conditions of atmospheric pollution when the measured concentrations of nitrogen dioxide and ammonia in the atmosphere exceed the corresponding maximum one-time TLV (Fig. 1).
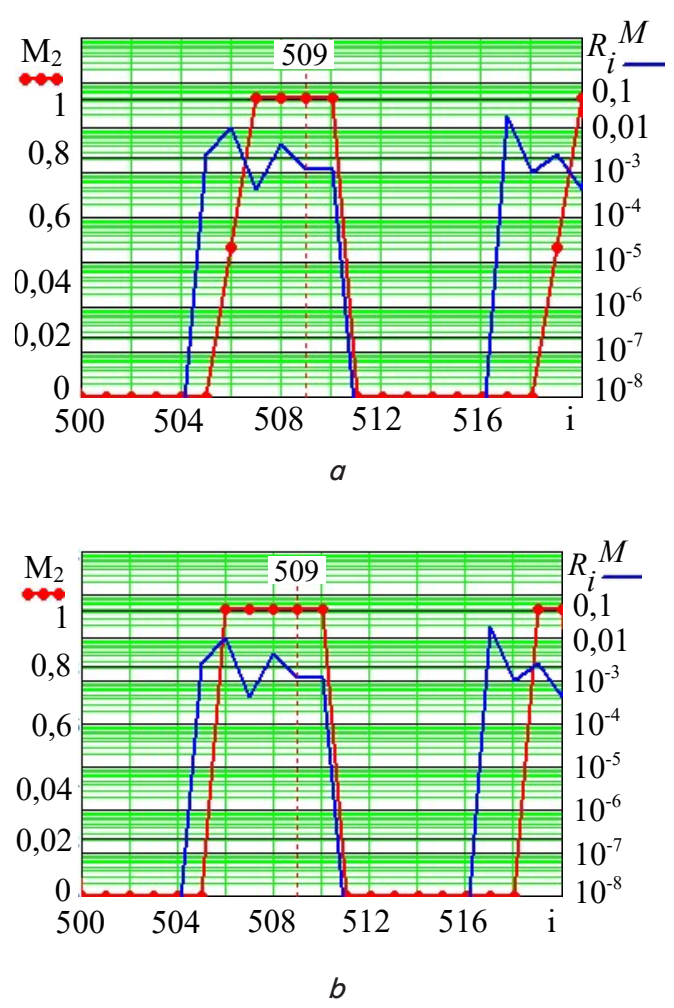

Fig. 3. The dynamics of discrete values of the combined risk of instantaneous action (2) and the CRLR window measure (4)

for two values of the $w$ parameter: $a-w=1 ; b-w=0$

\section{Discussion of results from testing the proposed method}

The experimental data that are given in Fig. 1 clearly illustrate that it is not possible to detect air pollution states dangerous for people based on the fact that the current concentrations of the pollutants exceed their maximum one-time TLV. For example, the current concentrations of nitrogen dioxide and ammonia, measured at the test interval, in most cases do not exceed the maximum one-time TLV (red dots) set for them. In contrast, the levels of risk to public health that are corresponding to these measured concentrations of pollutants indicate a significant (on average, by $10^{5}$ times) excess over the level of the upper limit of the permissible individual risk (blue points). This confirms that the current world practice of assessing the dangers of AP is based on determining the risks to life and health. Therefore, the 
developed method for detecting the hazardous conditions of atmospheric pollution, based on the current values of the level of the combined risk of instantaneous action for a totality of pollutants, does not contradict world practice. It should be noted that the observed experimental excess of the level of the upper limit of permissible individual risk by $10^{5}$ times, can cause, for the average statistical individual or a group of people, a reliable disruption of normal life conditions, manifested in the loss of consciousness and other acute undesirable effects.

The capabilities of the RP method to show the hidden dynamics of the combined risk of instantaneous action for a group of pollutants in real time at the predefined pollution control point are illustrated in Fig. 2. In this case, black dots in Fig. 2 show the recurrent values in the restored dynamics of the current level of the combined risk of instantaneous action. White dots in Fig. 2 demonstrate values for the absence of such recurrence. Our analysis of the point representation, shown in Fig. 2, reveals that the restored dynamics of the combined risk of instantaneous action is generally not random but chaotic, and is characterized by clusters of the recurrent values of the combined risk levels. The identification of such clusters in real time makes it possible to detect dangerous conditions of the contaminated atmosphere based on a combination of harmful substances, as well as the levels of the combined risk of instantaneous action for the population at the predefined point of control. At the same time, the use of the CRLR window measure (4) in the proposed method makes it possible to detect, in real time, not only the dangerous conditions of the contaminated atmosphere but also, at the same time, to assess the probability of their occurrence (red curves in Fig. 3). To confirm this fact, Fig. 3 shows the discrete values of the level of combined risk (2) in real time and the CRLR measure (4) for a fragment of the test interval, including the time of count 509, associated with a dangerous event of the violation of the conditions of normal life (loss of consciousness) for a group of people in the open territory. According to the experimental data in Fig. 3, at the time of count 509, the level of the combined risk of instantaneous action to health was $10^{-3}$. In this case, the probability estimate (a CRLR measure) was equal to unity. For this case, the upper limit of the acceptable individual risk was $5,708 \cdot 10^{-8}$. This means that the proposed method has reliably identified the time associated with the presence of an unacceptable level of the combined risk of instantaneous action corresponding to the dangerous state of atmospheric pollution. In this case, it follows from the analysis of dependences in Fig. 3 that with some reduction in the allowable probability (a CRLR measure) and a reduction in the width of the averaging window in (4), the proposed method makes it possible not only to detect dangerous conditions of the contaminated atmosphere but also to pre-empt (to implement short-term forecast) their occurrence. For example, from analyzing experimental data in Fig. 3 , it follows that pre-empting the appearance of hazardous conditions of the contaminated atmosphere on the basis of the proposed method at the specified point of control (with a discrete measurement of the pollutants' concentrations at intervals of 6 hours) can be carried out in 18 hours. This interval of discrete measurement of the pollutants' concentration is implemented at existing stationary control posts in cities.

An important advantage of the proposed method's application is that it is based only on the measurements of the current concentrations of pollutants at points of control.
Given the current level of the development of AP measurements, these measurements can be performed with any time discreteness while ensuring the high accuracy and reliability of the measurement results. Therefore, in contrast to known methods that require a large amount of additional information about the conditions of measurements at the predefined point of control, the method described is universal and can be used for any point. This is because the proposed method is based on determining the hidden dynamics of the combined risk level, taking into consideration the impact of all factors at the point of control. In this case, the nature of the recurrence of values of the combined risk in the specified dynamic makes it possible to detect dangerous conditions of the contaminated atmosphere. The limitation of this study is the consideration of the limited number of pollutants in the experiment. However, this limitation can be addressed by increasing the number of pollutants of different hazard classes in the experiment. In addition, this study is limited to an experiment at one point of control of the city with an average level of air pollution. The elimination of the noted disadvantage is associated with the expansion of the geography of research to cover many points of control over different areas of industrial cities with different levels of AP.

This study could be advanced by tackling the limitations mentioned above.

\section{Conclusions}

1. We have developed a method for detecting, in real time, the hazardous conditions of contaminated air in urban areas for an arbitrary number of pollutants, which is based on restoring the hidden dynamics in the level of the combined risk of instantaneous action only by the current measurements of the concentration of pollutants at a point of control. No other data on current conditions are used at the control point. Therefore, the proposed method, in contrast to known analogs, is universal and can be used for any control points. In this case, the restored dynamics in the level of the combined risk of instantaneous action make it possible not only to identify dangerous conditions of contaminated atmospheric air but also, based on the current recurrence of the combined risk levels, to assess the probability of detecting and predicting the dangerous levels of the combined risk of instantaneous action in real time at the predefined point of control. The use of the developed method at several control points in an arbitrary area would make it possible to determine the space-time distribution of the levels of the combined risk of instantaneous action relating to atmospheric pollution for the population of the territory.

2. Experimental measurements of the concentration of formaldehyde, ammonia, and nitrogen dioxide in contaminated atmospheric air at the point of control in one of the industrial cities with an air pollution level of about 37 units, the AQI scale (USA), have been performed. Based on the measurements obtained, the proposed method was tested, which confirmed its overall performance under experimental conditions. It has been established that the level of the combined risk of instantaneous action at the time of count 509, associated with an actual dangerous event (the loss of consciousness by a group of people), is about $10^{-3}$ with a single probability of this level of risk. This means that a given level of the combined risk of instantaneous action can be considered to be almost a valid value, exceeding by about $10^{5}$ times 
the level of the recalculated upper limit of the permissible individual risk. It is noted that with a decrease in the probability for the level of the combined risk of instantaneous action and the width of the averaging window, it is possible to predict the occurrence of dangerous levels of combined risk. The example of the considered actual dangerous event has experimentally established that the maximum forecast time of the dangerous level of the combined risk does not exceed 18 hours. This forecast time is based on a discrete measurement of pollutant concentrations at intervals of 6 hours. A given measurement regime is implemented at the existing city air pollution control posts.

\section{References}

1. Egondi, T., Kyobutungi, C., Ng, N., Muindi, K., Oti, S., Vijver, S. et. al (2013). Community Perceptions of Air Pollution and Related Health Risks in Nairobi Slums. International Journal of Environmental Research and Public Health, 10(10), 4851-4868. doi: https://doi.org/10.3390/ijerph10104851

2. Argyropoulos, C. D., Ashraf, A. M., Markatos, N. C., Kakosimos, K. E. (2017). Mathematical modelling and computer simulation of toxic gas building infiltration. Process Safety and Environmental Protection, 111, 687-700. doi: https://doi.org/10.1016/ j.psep.2017.08.038

3. Sorek-Hamer, M., Chatfield, R., Liu, Y. (2020). Review: Strategies for using satellite-based products in modeling PM2.5 and shortterm pollution episodes. Environment International, 144, 106057. doi: https://doi.org/10.1016/j.envint.2020.106057

4. Zou, B., Wilson, J. G., Zhan, F. B., Zeng, Y. (2009). Air pollution exposure assessment methods utilized in epidemiological studies. Journal of Environmental Monitoring, 11 (3), 475. doi: https://doi.org/10.1039/b813889c

5. Beckx, C., Int Panis, L., Arentze, T., Janssens, D., Torfs, R., Broekx, S., Wets, G. (2009). A dynamic activity-based population modelling approach to evaluate exposure to air pollution: Methods and application to a Dutch urban area. Environmental Impact Assessment Review, 29 (3), 179-185. doi: https://doi.org/10.1016/j.eiar.2008.10.001

6. Bell, M. L., Ebisu, K., Belanger, K. (2007). Ambient Air Pollution and Low Birth Weight in Connecticut and Massachusetts. Environmental Health Perspectives, 115 (7), 1118-1124. doi: https://doi.org/10.1289/ehp.9759

7. Ballester, F. (2002). The EMECAM project: a multicentre study on air pollution and mortality in Spain: combined results for particulates and for sulfur dioxide. Occupational and Environmental Medicine, 59 (5), 300-308. doi: https://doi.org/10.1136/ oem.59.5.300

8. Kustov, M. V., Kalugin, V. D., Tutunik, V. V., Tarakhno, E. V. (2019). Physicochemical principles of the technology of modified pyrotechnic compositions to reduce the chemical pollution of the atmosphere. Voprosy Khimii i Khimicheskoi Tekhnologii, 1, 92-99. doi: https://doi.org/10.32434/0321-4095-2019-122-1-92-99

9. Pascual, M., Ellner, S. P. (2000). Linking ecological patterns to environmental forcing via nonlinear time series models. Ecology, 81 (10), 2767-2780. doi: https://doi.org/10.1890/0012-9658(2000)081[2767:leptef]2.0.co;2

10. Parrott, L. (2004). Analysis of simulated long-term ecosystem dynamics using visual recurrence analysis. Ecological Complexity, 1 (2), 111-125. doi: https://doi.org/10.1016/j.ecocom.2004.01.002

11. Proulx, R. (2007). Ecological complexity for unifying ecological theory across scales: A field ecologist's perspective. Ecological Complexity, 4 (3), 85-92. doi: https://doi.org/10.1016/j.ecocom.2007.03.003

12. Eckmann, J.-P., Kamphorst, S. O., Ruelle, D. (1987). Recurrence Plots of Dynamical Systems. Europhysics Letters (EPL), 4 (9), 973-977. doi: https://doi.org/10.1209/0295-5075/4/9/004

13. Webber, Jr. C. L., Zbilut, J. P. (2005). Recurrence quantification analysis of nonlinear dynamical systems. Tutorials in contemporary nonlinear methods for the behavioral sciences, 26.

14. Pospelov, B., Andronov, V., Rybka, E., Meleshchenko, R., Borodych, P. (2018). Studying the recurrent diagrams of carbon monoxide concentration at early ignitions in premises. Eastern-European Journal of Enterprise Technologies, 3 (9 (93)), 34-40. doi: https:// doi.org/10.15587/1729-4061.2018.133127

15. Turcotte, D. L. (1977). Fractals and chaos in geology and geophysics. Cambridge University Press. doi: https://doi.org/10.1017/ cbo9781139174695

16. Pospelov, B., Rybka, E., Meleshchenko, R., Borodych, P., Gornostal, S. (2019). Development of the method for rapid detection of hazardous atmospheric pollution of cities with the help of recurrence measures. Eastern-European Journal of Enterprise Technologies, 1 (10 (97)), 29-35. doi: https://doi.org/10.15587/1729-4061.2019.155027

17. Pospelov, B., Andronov, V., Rybka, E., Krainiukov, O., Karpets, K., Pirohov, O. et. al. (2019). Development of the correlation method for operative detection of recurrent states. Eastern-European Journal of Enterprise Technologies, 6 (4 (102)), 39-46. doi: https://doi.org/ 10.15587/1729-4061.2019.187252

18. Pospelov, B., Rybka, E., Togobytska, V., Meleshchenko, R., Danchenko, Y., Butenko, T. et. al. (2019). Construction of the method for semi-adaptive threshold scaling transformation when computing recurrent plots. Eastern-European Journal of Enterprise Technologies, 4 (10 (100)), 22-29. doi: https://doi.org/10.15587/1729-4061.2019.176579

19. Pospelov, B., Rybka, E., Meleshchenko, R., Krainiukov, O., Harbuz, S., Bezuhla, Y. et. al. (2020). Use of uncertainty function for identification of hazardous states of atmospheric pollution vector. Eastern-European Journal of Enterprise Technologies, 2 (10 (104)), 6-12. doi: https://doi.org/10.15587/1729-4061.2020.200140

20. Pospelov, B., Andronov, V., Rybka, E., Krainiukov, O., Maksymenko, N., Meleshchenko, R. et. al. (2020). Mathematical model of determining a risk to the human health along with the detection of hazardous states of urban atmosphere pollution based on 
measuring the current concentrations of pollutants. Eastern-European Journal of Enterprise Technologies, 4 (10 (106)), 37-44. doi: https://doi.org/10.15587/1729-4061.2020.210059

21. Ferrante, M., Fiore, M., Copat, C., Morina, S., Ledda, C., Mauceri, C., \& Oliveri Conti, G. (2015). Air Pollution in High-Risk SitesRisk Analysis and Health Impact. Current Air Quality Issues. doi: https://doi.org/10.5772/60345

22. Naydenko, V. V., Gubanov, L. N., Kosarikov, A. N., Afanas'eva, I. M., Ivanov, A. V. (2003). Ekologo-ekonomicheskiy monitoring okruzhayushchey sredy. Nizhniy Novgorod, 186.

23. Leonovich, E. I., Skorobogataya, I. V. (2019). Otsenka riska dlya zhizni i zdorov'ya naseleniya ot vozdeystviya zagryaznyayushchih veshchestv v atmosfernom vozduhe. Gigienicheskie pokazateli urovnya zagryazneniya atmosfery. Minsk, 48.

24. Poulsen, A., Jomaas, G. (2011). Experimental Study on the Burning Behavior of Pool Fires in Rooms with Different Wall Linings. Fire Technology, 48 (2), 419-439. doi: https://doi.org/10.1007/s10694-011-0230-0

25. Pospelov, B., Meleshchenko, R., Krainiukov, O., Karpets, K., Petukhova, O., Bezuhla, Y. et. al. (2020). A method for preventing the emergency resulting from fires in the premises through operative control over a gas medium. Eastern-European Journal of Enterprise Technologies, 1 (10 (103)), 6-13. doi: https://doi.org/10.15587/1729-4061.2020.194009

26. Kondratenko, O. M., Vambol, S. O., Strokov, O. P., Avramenko, A. M. (2015). Mathematical model of the efficiency of diesel particulate matter filter. Naukovyi Visnyk Natsionalnoho Hirnychoho Universytetu, 6, 55-61.

27. Semko, A. N., Beskrovnaya, M. V., Vinogradov, S. A., Hritsina, I. N., Yagudina, N. I. (2014). The usage of high speed impulse liquid jets for putting out gas blowouts. Journal of Theoretical and Applied Mechanics, 52 (3), 655-664.

28. Otrosh, Y., Semkiv, O., Rybka, E., Kovalov, A. (2019). About need of calculations for the steel framework building in temperature influences conditions. IOP Conference Series: Materials Science and Engineering, 708, 012065. doi: https://doi.org/10.1088/ $1757-899 x / 708 / 1 / 012065$

29. Vambol, S., Vambol, V., Kondratenko, O., Koloskov, V., Suchikova, Y. (2018). Substantiation of expedience of application of hightemperature utilization of used tires for liquefied methane production. Journal of Achievements in Materials and Manufacturing Engineering, 2 (87), 77-84. doi: https://doi.org/10.5604/01.3001.0012.2830

30. Loboichenko, V. M., Vasyukov, A. E., Tishakova, T. S. (2017). Investigations of Mineralization of Water Bodies on the Example of River Waters of Ukraine. Asian Journal of Water, Environment and Pollution, 14 (4), 37-41. doi: https://doi.org/10.3233/ajw-170035 